\title{
A novel dual-adhesive and bioactive hydrogel activated by bioglass for wound healing
}

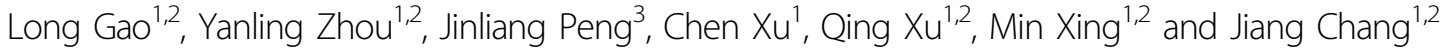

\begin{abstract}
Dual adhesiveness to tissue and implant biomaterials and bioactivity to stimulate tissue regeneration are interesting properties for developing new generations of tissue-repairing hydrogels with potential new clinical applications. In this study, we developed a unique bioglass (BG)/oxidized sodium alginate (OSA) composite hydrogel with adipic acid dihydrazide ( $A D H)$-modified $\gamma$-polyglutamic acid ( $\gamma$-PGA) as the cross-linking agent, in which the BG plays a multifunctional role to endow the hydrogel with both dual-adhesive and bioactive properties. On one hand, the BG could improve the tissue-bonding strength by providing an alkaline microenvironment to stimulate the bond formation between OSA and the amino groups on the surrounding tissues. On the other hand, BG endows the hydrogel with adhesiveness to implantable materials by releasing $\mathrm{Ca}$ ions, which might chelate with the carboxyl groups of the hydrogel matrix. In addition, the composite hydrogel showed excellent bioactivity to promote vascularization and accelerate tissue regeneration. This study demonstrates that a multifunctional hydrogel can be designed by utilizing the multifunctional ions released from silicate BG, and the BG/OSA hydrogel shows good potential as an adhesive and bioactive material for woundhealing applications.
\end{abstract}

\section{Introduction}

Hydrogels are polymeric materials with threedimensional cross-linked networks that could act as a barrier to prevent bacterial infection and create a suitable microenvironment for tissue regeneration ${ }^{1-3}$. Hydrogels with high adhesiveness to tissues may bind tissues together and prevent the scar tissue formation that is induced by conventional sutures ${ }^{4}$. Furthermore, hydrogels with adhesiveness to materials may be used as biocompatible coatings on implantable materials for fixing implants ${ }^{5}$, monitoring human activity ${ }^{6}$, or avoiding implant dislocation during or after surgery ${ }^{7}$. However, preparing a hydrogel with adhesiveness to both tissues and implant materials remains a challenge. The tissue-adhesion

\footnotetext{
Correspondence: Jiang Chang (jchang@mail.sic.ac.cn)

${ }^{1}$ State Key Laboratory of High Performance Ceramics and Superfine

Microstructure, Shanghai Institute of Ceramics, Chinese Academy of Sciences, 1295 Dingxi Road, Shanghai 200050, China

${ }^{2}$ Center of Materials Science and Optoelectronics Engineering, University of Chinese Academy of Sciences, 19 Yuquan Road, Beijing 100049, China

Full list of author information is available at the end of the article.
}

capability of a hydrogel is attributed to the chemical/ physical linkages formed between the hydrogel and the surrounding tissues ${ }^{8,9}$. Because there are a large number of amino groups on the surface of tissues, an amide linkage is one of the most commonly used linkages between adhesive materials and tissue when the hydrogel contains aldehyde groups, which can link with the amino groups on the surrounding tissues ${ }^{4,10}$. It is known that the formation of amide and imine bonds between adhesive hydrogels and tissues requires an alkaline environment ${ }^{11,12}$. However, the $\mathrm{pH}$ value in the wound area is generally in the weakly acidic or neutral range $(5.4-7.4)^{13}$, or even acidic in the case of cancer tissue ${ }^{14}$, which will result in a lack of binding strength to tissues. Therefore, a weak alkaline environment is of great importance for the improvement of the adhesive strength of amide linkage-based adhesive hydrogels to tissues. The adhesion strength of the hydrogels to materials may be affected by some factors ${ }^{5,15,16}$, and an improvement in the viscosity of the hydrogels may result in stronger adhesion to the materials ${ }^{17}$. 
In addition to adhesiveness to both tissues and implant materials, the bioactivity of hydrogels is a critical factor that may significantly affect the wound-healing process. A bioactive hydrogel may be able to stimulate cell migration $^{18}$, proliferation ${ }^{19}$, and differentiation ${ }^{20}$, and enhance blood vessel formation during the woundhealing process ${ }^{21}$, so that the hydrogel can significantly reduce the healing time ${ }^{21-23}$. Currently, hydrogels with bioactivity are mainly obtained by the addition of growth factors $^{24}$, which still faces challenges in clinical applications, such as their controlled delivery and activity maintenance.

Sodium alginate (SA) is a known biomaterial that can form hydrogels through divalent ion cross-linking and has good biocompatibility in biomedical applications ${ }^{15,18,19}$. The functional groups of the alginate molecule chains can be easily oxidized for imine formation ${ }^{11,25}$, which may be utilized for the adhesive reaction with tissues. It is also known that the formation of imine bonds is favored by an alkaline environment ${ }^{12}$. Bioglass (BG) is an inorganic biomaterial that has shown the bioactivities of acceleration of vascularization and enhancement of tissue regeneration through the release of bioactive $\mathrm{Si}$ ions ${ }^{21,26}$. Furthermore, ion exchange on the BG surface could create an alkaline environment and release $\mathrm{Ca}^{2+}$ into aqueous solution ${ }^{27}$, where the $\mathrm{Ca}^{2+}$ can also be involved in chelation with the carboxyl groups in the alginate polymer chains to improve the adhesion of the hydrogels onto materials ${ }^{28}$. Therefore, our hypothesis is that if BG is added into an alginate solution, it may result in the formation of bioactive and adhesive hydrogels due to the multifunctional roles of BG. One role is the rapid ion exchange on the BG surface, which creates an alkaline environment to accelerate the formation of an imine bond for enhanced adhesiveness to tissues. The second role is the release of $\mathrm{Ca}^{2+}$, which physically cross-links the polymer chains via chelation between $\mathrm{Ca}^{2+}$ and carboxyl groups. This highly reversible cross-link improves the viscosity and cohesion of the hydrogels, resulting in an increased adhesiveness to implantable materials. The last role of the BG is the release of $\mathrm{Si}$ ions, which endows the hydrogel with the capacity to enhance vascularization and tissue regeneration.

Based on these considerations, we designed a unique BG/OSA composite hydrogel with adipic acid dihydrazide (ADH)-modified $\gamma$-polyglutamic acid ( $\gamma$-PGA) as the cross-linking agent, which not only has good adhesiveness to tissues but is also adhesive to different implantable materials. This dual adhesiveness to both tissues and materials was evaluated, and the application of the hydrogel for skin wound healing was also evaluated in vivo in two different wound healing mouse models to confirm the adhesiveness and bioactivity of the hydrogel.

\section{Materials and methods}

\section{Preparation of the hydrogels}

First, oxidized sodium alginate (OSA) was obtained by the periodate oxidation of SA as reported ${ }^{11,29}$. OSA was obtained by adding $1.0 \mathrm{~g}$ of SA (Sigma Aldrich, USA) and $0.054 \mathrm{~g}$ of sodium periodate (Sigma Aldrich, USA) to $100 \mathrm{ml}$ of distilled water in the dark. The reaction was terminated by the addition of $1.5 \mathrm{ml}$ of ethylene glycol after $2.0 \mathrm{~h}$. The cross-linking agent ADH-modified $\gamma$-PGA was obtained by the grafting reaction between $A D H$ and $\gamma$-PGA as reported ${ }^{29,30}$. Briefly, $0.57 \mathrm{~g}$ of ADH (Aladdin, China), $1.0 \mathrm{~g}$ of $\gamma$-PGA (Beijing HWRK chem Co. LTD, China), and $\mathrm{N}$-hydroxysuccinimide (Adamas-beta, China) were dissolved in $100 \mathrm{ml}$ of water. Then, the $\mathrm{pH}$ was adjusted to $5.0 \sim 5.5$, and the reaction lasted for $12.0 \mathrm{~h}$ after the addition of $0.60 \mathrm{~g}$ of 1-ethyl-3-(3'-dimethylaminopropyl) carbodiimide (Adamas-beta, China). The resulting OSA and ADH-modified $\gamma$-PGA was then purified via dialysis (MWCO 30000), followed by freezedrying.

The BG/OSA composite hydrogel was prepared as follows. First, $1.0 \mathrm{ml}$ of ADH-modified $\gamma$-PGA solution (20 wt\%) was homogeneously dispersed into $1.0 \mathrm{ml}$ of OSA solution (20 wt\%). Then, $50 \mathrm{mg}$ of glutamic acid (Aladdin, China) was dissolved in the solution, and the hydrogel gradually formed. The BG/OSA composite hydrogel was obtained by the addition of 45S5 BG powders $(0.5 \%, 1.0 \%$, or $1.5 \%$ by weight) (Kunshan Overseas Chinese Science and Technology New Material Co. LTD, China) into the OSA solution and mixing well before the $\gamma$-PGA solution was added. The gelling solution was gently stirred until the hydrogel formed. The $\mathrm{pH}$ of the hydrogels was measured with a $\mathrm{pH}$ meter (INESA PHSJ$4 \mathrm{~F})$. The gelling time and ion release of the hydrogels were clarified as in our previous report ${ }^{15,19}$. A $1.0 \%$ calcium carbonate (CA)/OSA hydrogel was prepared by adding $1.0 \% \mathrm{CA}$ powder into the gelling solution instead of BG according to the same procedure as described above for the preparation of the BG/OSA hydrogels. A $1.0 \%$ $\mathrm{Na}_{2} \mathrm{CO}_{3} / \mathrm{OSA}$ hydrogel was prepared by adding $1.0 \%$ $\mathrm{Na}_{2} \mathrm{CO}_{3}$ particles to the OSA/ $\gamma$-PGA gelling solution. $\mathrm{CaCl}_{2} / \mathrm{OSA}$ hydrogels were prepared by adding $0.1 \mathrm{ml}$ of $\mathrm{CaCl}_{2}$ solution $(5 \mathrm{mg} / \mathrm{ml})$ to $1.0 \mathrm{ml}$ of the OSA $/ \gamma-\mathrm{PGA}$ gelling solution.

\section{Characterization of the adhesiveness of the hydrogels}

The adhesive strengths on tissues were measured by lap-shear strength tests on porcine skin ${ }^{31}$. The adhesiveness of the BG/OSA hydrogel and quantitative analysis of the adhesive strength were carried out according to the previous method $^{21}$. As shown in Fig. 2b, the test method of tissue adhesion is illustrated in the schematic diagram. First, fresh porcine skin was cleaned as reported ${ }^{32}$ and cut into strips of size $35 \mathrm{~mm} \times 10 \mathrm{~mm}$. Then, $100 \mu \mathrm{l}$ of freshly 
prepared gelling solution was applied to one piece, and another piece was brought in contact with the first piece to form a contact area of $10 \times 10 \mathrm{~mm}^{2}$. After applying a $50 \mathrm{~g} / \mathrm{cm}^{2}$ load for $4.0 \mathrm{~h}$, the adhesion strength could be measured through a universal testing machine (Shimadzu Corporation, Japan).

The adhesive strengths of the hydrogels on the implant materials were also measured by lap-shear strength tests as reported in dry conditions ${ }^{17}$. Some commonly used implant materials, including silicone, titanium alloy, tricalcium phosphate (TCP), and porous TCP, were cut into strips of size $10 \mathrm{~mm} \times 10 \mathrm{~mm}$. Then, $100 \mu \mathrm{l}$ of freshly prepared gelling solution was applied to one piece of implant material, and then another piece was brought in contact for $4.0 \mathrm{~h}$. The adhesion strengths were measured as previously described.

Rheology tests were performed using a compact Anton Paar modular in oscillation mode. The viscosities of the newly prepared hydrogels were measured, and all viscosities were obtained by a frequency sweep with a fixed strain of $1.0 \%$. To further explore possible mechanisms, the viscosities and adhesion strengths of the 1.0\%-BG/ OSA hydrogel, the $1.0 \%$-CA/OSA hydrogel, the $0.5 \mathrm{mg} / \mathrm{ml}$ $\mathrm{CaCl}_{2} / \mathrm{OSA}$ hydrogel, the $1.0 \%-\mathrm{Na}_{2} \mathrm{CO}_{3} / \mathrm{OSA}$ hydrogels, and the OSA hydrogel on materials or porcine skins were further compared.

\section{In vitro cell culture experiments}

Human umbilical vein endothelial cells (HUVECs) and human dermal fibroblasts (HDFs) play an important role in the wound-healing process. In this study, HDFs and HUVECs were chosen to evaluate the biological effects of the BG/OSA hydrogels. HDFs were isolated from the superficial layer of adult human skin and cultured in DMEM-high (Gibco) plus 10\% (v/v) fetal bovine serum (FBS) and $1 \%(\mathrm{v} / \mathrm{v})$ penicillin-streptomycin (P/S). HUVECs were separated from the human umbilical cord vein and cultured in ECM (ScienCell, USA) supplemented with $5 \%(\mathrm{v} / \mathrm{v})$ FBS, $1 \%(\mathrm{v} / \mathrm{v}) \mathrm{P} / \mathrm{S}$ and $1 \%(\mathrm{v} / \mathrm{v})$ endothelial cell growth supplement (ECGS). To determine the cytocompatibility of the composite hydrogel, a $100 \mu \mathrm{l}$ extract of the 1.0\%-BG/OSA hydrogel or the pure OSA hydrogel was added to 96-well plates, and HUVECs or HDFs were seeded at a density of $4 \times 10^{4}$ cells $/ \mathrm{cm}^{2}$. There were no hydrogel extracts in the control groups. A quantitative assessment of cell viability was performed using the Cell Counting Kit (CCK)-8 assay (Beyotime) as we previously reported after culturing for 1,3 , and 7 days $^{18}$. The expression levels of the vascular endothelial growth factor (VEGF) genes of the HDFs and HUVECs were determined by qRT-PCR analysis ${ }^{33}$ on day 3 of culture. Briefly, the total RNA was extracted from cells using TRIzol reagent (Invitrogen, USA) according to the manufacturer's protocol. After determination of the RNA concentration and
cDNA synthesis, real-time PCR was carried out using a Step One Plus Real-Time PCR System (Applied Biosystems, USA). The primer sequences used for qRT-PCR analysis were forward primer sequence $\left(5^{\prime}-3^{\prime}\right)$ : TATGCGGATCAAACCTCACCA and reverse primer sequence $\left(5^{\prime}-3^{\prime}\right)$ : CACAGGGATTTTTCTTGTCTTGCT.

\section{Wound closure in vivo}

The protocols were approved by the Shanghai Jiao Tong University animal care and use committee guidelines. The effects of the hydrogel on wound closure was evaluated in a mouse model as reported ${ }^{34}$. Briefly, a drop of $1.0 \%$-BG/ OSA or pure OSA gelling solution was used on the wound edge. Then, the two wound edges were brought in contact for $1 \mathrm{~min}$.

\section{Chronic wound healing in vivo}

Female nude mice (BALB/c, 6-8 weeks, $n=27)$ were randomly separated into three groups. After anesthesia with tribromoethanol $(1.2 \%, 0.02 \mathrm{ml} / \mathrm{g})$, a full-thickness excision $10 \mathrm{~mm}$ in diameter on each mouse's back was performed. For the OSA or $1.0 \%$-BG/OSA hydrogel groups, hydrogel cylinders $10 \mathrm{~mm}$ in diameter were used on the wound site. For the Blank group, the animals without any treatment were used as a negative control. Then, $100 \mu \mathrm{l}$ of Depo Medrol (20 mg/kg BW, Pfizer) was subcutaneously injected into all mice to delay wound healing. Seven, 14, and 21 days later, the mice were killed, and the tissue explants at the wound site were harvested. Histological and immunohistochemical analyses were performed as in our previous report ${ }^{21}$.

\section{Results}

\section{Preparation of the BG/OSA hydrogels}

As shown in Fig. 1a, the homogeneous composite hydrogels were obtained with different amounts of BG. $\mathrm{ADH}$-modified $\gamma$-PGA is a biocompatible cross-linking agent that constitutes OSA gels ${ }^{11,29}$. However, the reaction is too rapid, and there is hardly enough time for the operation. The injection of the OSA hydrogel is nearly

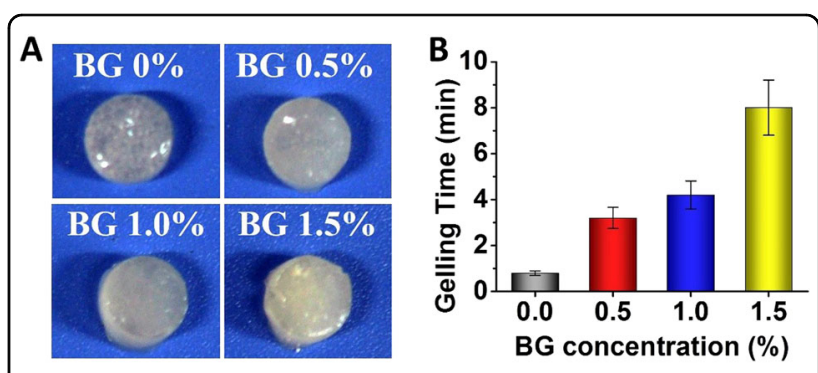

Fig. 1 The obtained BG/OSA hydrogels. a Optical images of the OSA and BG/OSA hydrogels. $\mathbf{b}$ Gelling time of the hydrogels with different amounts of BG 

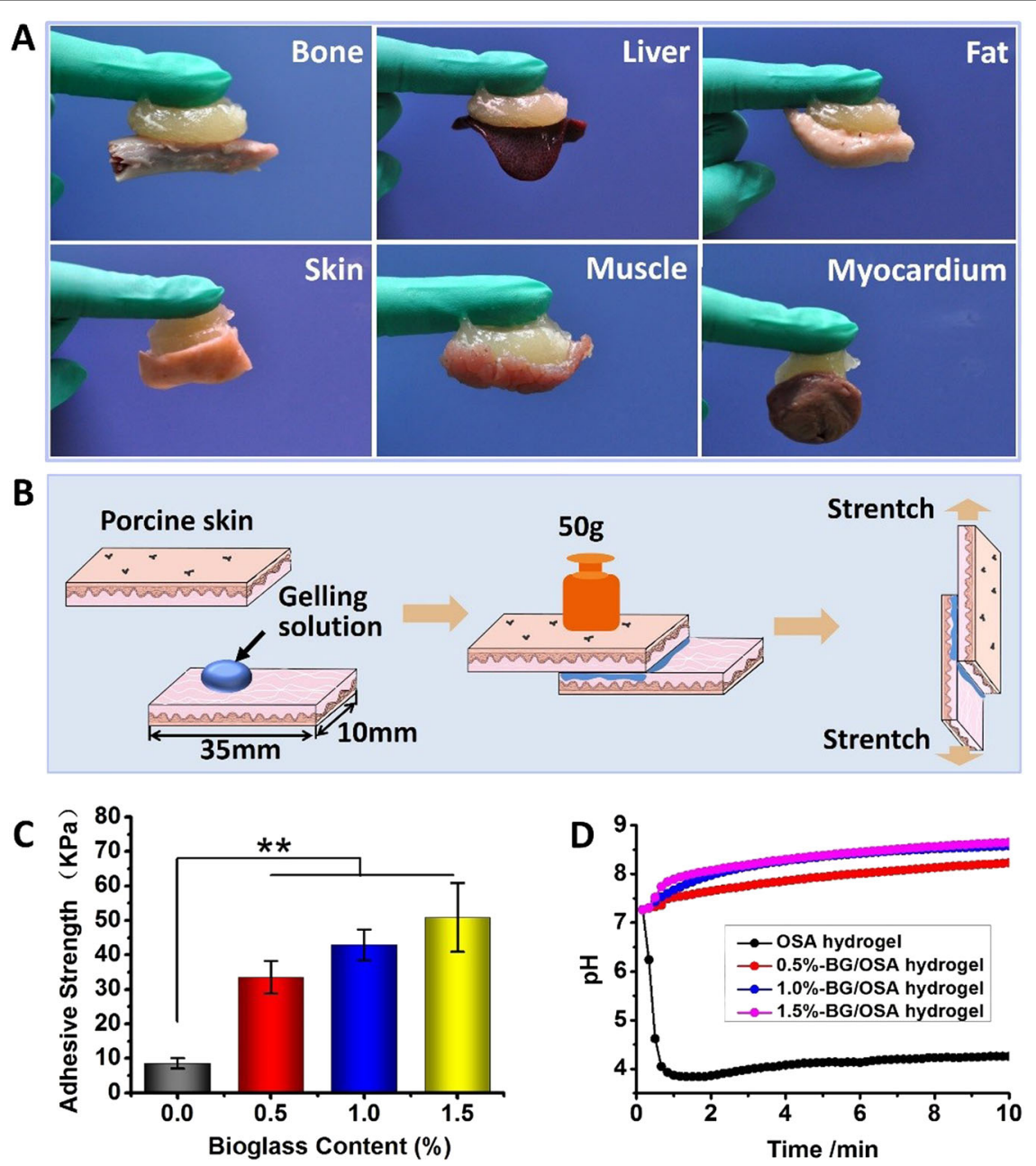

Fig. 2 BG endowed the hydrogels with adhesive properties to various tissues. a Adhesion exhibition of the BG/OSA hydrogel for pig tissues, including fat, liver, bone, skin, muscle, and myocardium. $\mathbf{b}$ Schematic of the method used to measure the bonding strength of the hydrogels on porcine skin. $\mathbf{c}$ Adhesive strengths of the OSA hydrogel and BG/OSA hydrogel on two pieces of porcine skin with the addition of different amounts of BG measured by lap-shear strength tests. $\mathbf{d}$ The $\mathrm{pH}$ values of the hydrogels

impossible unless a dual syringe/syringe is used ${ }^{29,35}$. In this study, we attempted to prolong the gelling time by changing the amount of BG. The gelling time increased with an increase in BG content (Fig. 1b). The gelling time ranged from $<1 \mathrm{~min}$ for the OSA hydrogel without BG to 8.0 min for the BG/OSA hydrogel with $1.5 \%$ BG. It is known that the cross-linking reaction of OSA and ADHmodified PGA is fast in weakly acidic environments ${ }^{26}$. Before the addition of BG, the hydrogel solution was acidic (as shown in Fig. 2d), and the gelling reaction was rapid. After the addition of $\mathrm{BG}$, the $\mathrm{pH}$ of the hydrogel solution became weakly alkaline, which resulted in an increase in the gelation time.

\section{Dual adhesion}

Tissue adhesion is an essential factor for the practical applications of tissue adhesives ${ }^{4}$, wound dressing ${ }^{36}$, and wearable devices ${ }^{37}$. The adhesive properties of the composite hydrogels with various biological tissues were explored, and the results are given in Fig. 2a. Biological tissues, including the skin, liver, fat, bone, muscle, and myocardium, could easily adhere to the $1.0 \%$-BG/OSA hydrogel. Figure $2 \mathrm{c}$ shows the adhesive strength of the different hydrogels to different tissues, in which the adhesive strengths of the BG/OSA hydrogels to skin tissues were significantly higher than those of the OSA hydrogel. As shown in Fig. $2 \mathrm{~d}$, the $\mathrm{pH}$ value of the hydrogels increased gradually with the increase in BG amount in the hydrogels, indicating that the creation of an alkaline environment by BG addition played an important role in regulating hydrogel adhesiveness. As shown in Supplementary Fig. S1A, by comparison of the adhesiveness of the BG/OSA hydrogels to skin tissue with that of the calcium carbonate (CA)/OSA composite hydrogel, 

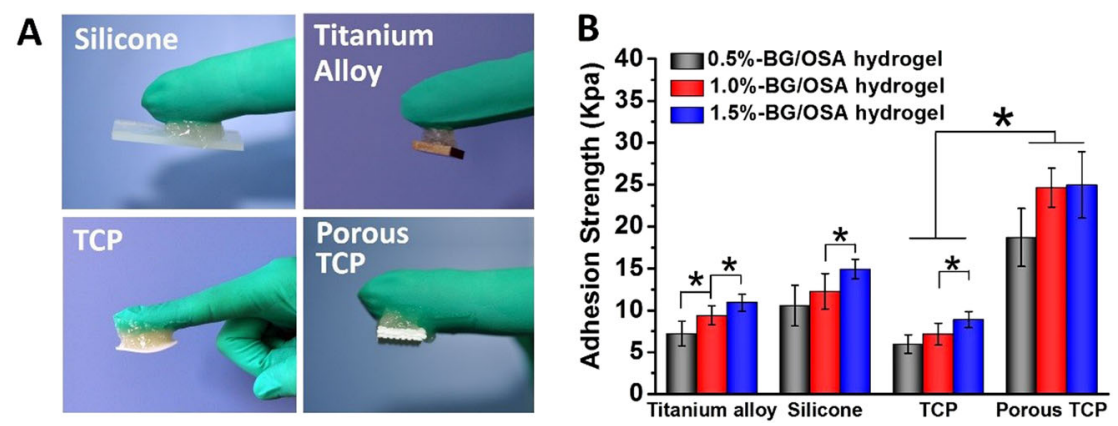

Fig. 3 BG endowed the hydrogel with adhesive properties for implant materials. a Adhesion of the BG/OSA hydrogel on different implant materials. $\mathbf{b}$ Adhesion strength of the BG/OSA hydrogel on silicone, titanium alloy, TCP, and a porous TCP scaffold measured by lap-shear strength tests under dry conditions

sodium carbonate/OSA composite hydrogel, and the hydrogel prepared with calcium chloride solution $\left(\mathrm{CaCl}_{2} /\right.$ OSA hydrogel), we found that although the CA/OSA hydrogel showed slightly improved adhesiveness to the skin compared with the OSA hydrogel, the CA/OSA hydrogel still showed significantly lower adhesiveness than that of the BG/OSA hydrogel. The $\mathrm{CaCl}_{2} / \mathrm{OSA}$ hydrogel and sodium carbonate/OSA hydrogel also showed similar adhesiveness to skin tissue as the pure OSA hydrogel. The $\mathrm{pH}$ measurements of the different hydrogels indicated that, compared with the BG/OSA hydrogel, the other hydrogels showed acidic or neutral $\mathrm{pH}$ values (Supplementary Fig. S1B), except for the $\mathrm{Na}_{2} \mathrm{CO}_{3} /$ OSA hydrogels, which showed a slightly alkaline $\mathrm{pH}$ at the beginning of gel formation, which then decreased to neutral with time.

In addition to the measurements of the adhesive strength between the hydrogels and different tissues, the adhesiveness of the BG/OSA hydrogel to different material surfaces was also evaluated. As shown in Fig. 3a, some commonly used implantable materials, including silicone, titanium alloy, and TCP, were employed to evaluate the adhesion of the BG/OSA hydrogel on the material's surface. The BG/OSA hydrogel showed adhesion to all these materials, and this adhesion strength increased with the increase in the amount of BG in the hydrogel. Considering that the regulation of adhesiveness of hydrogels to materials might be regulated by the chelation between $\mathrm{Ca}$ ions and the carboxyl groups of the polymer chains of the hydrogels, we further explored the possible mechanisms by comparing the adhesiveness of the BG/OSA hydrogel with the CA/OSA composite hydrogel, sodium carbonate/OSA composite hydrogel, and the hydrogel prepared with $\mathrm{CaCl}_{2} / \mathrm{OSA}$, which showed similar adhesiveness to the pure OSA hydrogel (Supplementary Fig. S2). These results showed that the CA/OSA hydrogel showed comparable adhesiveness to the BG/OSA hydrogel, while the $\mathrm{CaCl}_{2} / \mathrm{OSA}$ hydrogel and sodium carbonate/OSA hydrogel showed lower adhesiveness comparable with the pure OSA hydrogel (Supplementary Fig. S2). It is known that the adhesiveness of hydrogels to materials is closely related to both the surface roughness of the material and the viscosity of the hydrogel ${ }^{28}$. As shown in Fig. 3b, the hydrogel showed better adhesion to porous TCP discs than to the smooth TCP discs. To further explore the adhesion mechanism of the BG/OSA hydrogel to materials, the viscosity of the hydrogels was also measured. As shown in Supplementary Fig. S3, the introduction of BG into the hydrogel resulted in a significant increase in the viscosity. To elucidate the possible mechanisms of the adhesiveness to the materials, the viscosities of the hydrogels with different compositions were measured. As shown in Supplementary Fig. S4, the BG/OSA and the CA/OSA hydrogels showed similar viscosities, which were significantly higher than those of the pure OSA, the $\mathrm{CaCl}_{2} / \mathrm{OSA}$, and the $\mathrm{Na}_{2} \mathrm{CO}_{3} / \mathrm{OSA}$ hydrogel.

\section{Cell proliferation and bioactivity in vitro}

BG is known as a bioactive material, and here in our study, it indeed endowed the composite hydrogels with bioactivity by releasing bioactive $\mathrm{Si}$ ions. As shown in Fig. 4a, there was a steady and sustained Si ion release. Figure $4 \mathrm{~b}$, c shows the effects of the BG/OSA hydrogel on the proliferation of HUVECs and HDFs. A stimulatory effect on the proliferation of HUVECs and HDFs in the BG/OSA hydrogel group was observed compared with the Blank group, but no significant differences were found between the OSA hydrogel group and the Blank group.

More importantly, as shown in Fig. 4d, RT-PCR analyses revealed that the expression levels of vascular endothelial growth factor (VEGF) in HUVECs and HDFs loaded with $1.0 \%$-BG/OSA hydrogels were significantly higher than those of the cells growing in the OSA hydrogel on day 3 after seeding. These results suggest that the BG/OSA hydrogel might be able to promote angiogenesis. 

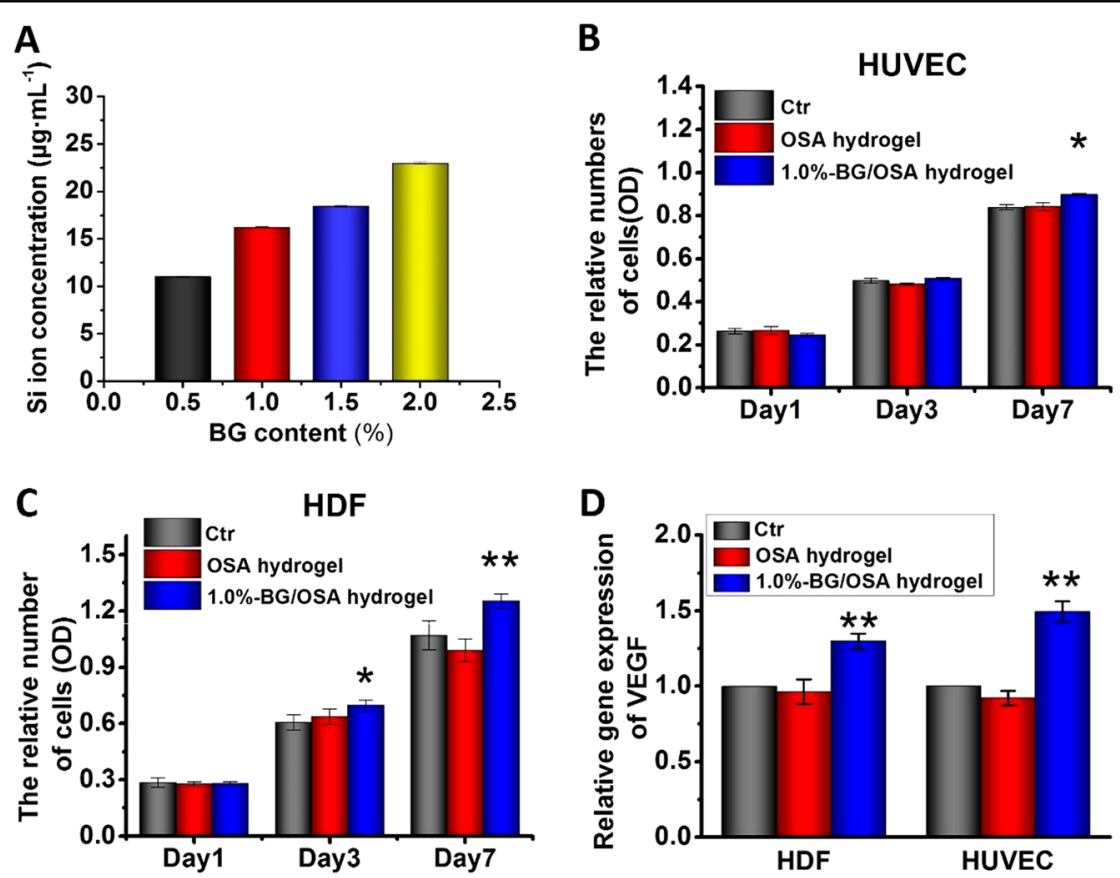

Fig. 4 Stimulation of cell proliferation and gene expression by bioactive BG/OSA composite hydrogels with ion-releasing properties. a Concentration of Si ions released from composite hydrogels with different amounts of BG. $\mathbf{b}, \mathbf{c}$ HUVEC and HDF proliferation and $\mathbf{d}$ VEGF gene expression on day 3

Effect of the BG/OSA hydrogel on wound closure in vivo

The adhesive performance of the composite hydrogel to skin wound healing was evaluated by a mouse wound closure model (Fig. 5a). As shown in Fig. 5b, 3 days after surgery, no wound leakages or infections were observed in the rat skins treated with the BG/OSA hydrogel. The obtained skin closures by the adhesive BG/OSA hydrogels, and the results were comparable with that of the traditional suture method. For the pure OSA hydrogel, which lacks enough tissue adhesion, the wound edges were not bonded by the hydrogel. The wound gaps and the blood scabs were as large as those in the Blank group. The H\&E staining shown in Fig. 5c clearly shows obvious epidermal gaps in the OSA hydrogel group and the Blank group, and the defect sites were covered with large blood scabs. In contrast, in the BG/OSA hydrogel group and the suturing group, intact epidermal layer formation was observed at the lacerated sites. The histological morphology at the repaired sites was similar to the adjacent normal tissue. This result suggests that wound closure is important for the healing process and that our adhesive hydrogel is as effective as traditional suture closure.

Effects of the BG/OSA hydrogel on wound healing in vivo

The effect of the bioactive BG/OSA composite hydrogel on the enhancement of wound healing was evaluated by a mouse chronic wound model. Wound closure and neovascularization in the BG/OSA hydrogel group, OSA hydrogel group, and Blank group were measured and compared. The gross observation photos of the wounds are given in Fig. 6a. They correspond to 7, 14, and 21 days after the different treatments. Almost no repair was observed in the Blank group after 7 days. In the OSA and BG/OSA hydrogel groups, the reduced wound area was obvious after 7 days. A light yellow color was observed, indicative of the remaining hydrogel on the top of the wound. At day 14, minimized wound area was observed in the BG/OSA group, the maximized blood scab area increased in the Blank group, and the OSA group had results between those of the other two groups. At day 21, the wound in the BG/OSA group was completely closed, and the Blank group still showed a large blood scab, while the OSA group had a result that was between those of the other two groups and showed a minimal blood scab. Quantification of wound sections revealed that the BG/ OSA hydrogel group exhibited the highest percent of wound closure, which was significantly different from the OSA hydrogel and Blank groups (Fig. 6c).

Figure $6 \mathrm{~b}$ represents the H\&E staining micrographs of all the groups. It is clear from the results that 7 days after surgery, there was already epidermis formation in the wound treated with the BG/OSA hydrogel, while no neoepidermis layer was observed in the other groups. 


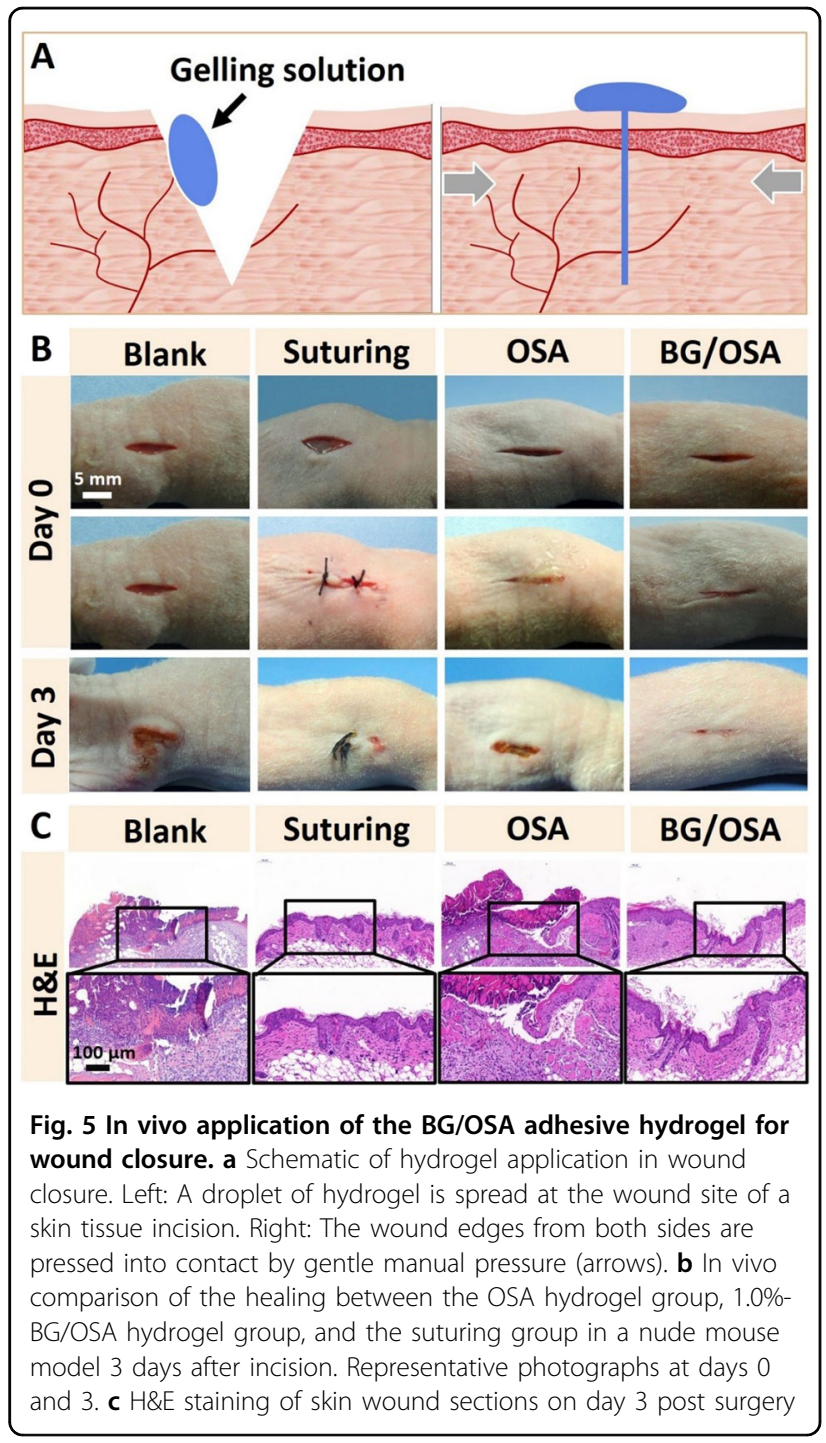

According to Fig. 7a, more CD 31-positive staining was observed in the BG/OSA hydrogel-treated wound area, whereas less $C D$ 31-positive staining was found in the OSA group and Blank group. The number of newly formed blood vessels in the BG/OSA group was significantly higher than that in the OSA and Blank groups 7, 14, and 21 days after surgery (Fig. 7b). Furthermore, it is interesting that on day 21 after treatment, the diameters of the newly formed blood vessels in the BG/OSA group were much larger than those in other groups (Fig. 7c).

Furthermore, the effects of the BG/OSA hydrogel to promote the process of wound healing was confirmed by immunohistochemical staining of collagen I and K14. As shown in Fig. 8a, 7 and 14 days post surgery, the staining intensity of collagen I in the newly formed skin in the BG/ OSA group was the strongest among the three groups, which indicates that the BG/OSA composite hydrogel stimulated collagen I secretion. On day 21 , the staining intensity of collagen $I$ in the BG/OSA group became weaker, which indicates that the healing process was almost complete. As shown in Fig. 8b, thicker and more uniform keratinized layers were found in the wounds treated with the BG/OSA hydrogel. This finding reveals that the composite hydrogel may stimulate the migration and proliferation of keratinocytes during wound healing.

\section{Discussion}

Moisture maintenance is necessary for the management of wounds. The hydrogel dressings could not only provide a moist environment but could also provide other multiple functions, such as anti-infective, antioxidative, and free radical scavenging capacities to promote wound healing ${ }^{38-40}$. However, most hydrogels do not have the ability to provide wound occlusion and stimulate blood vessel formation, which are important for chronic wound healing. In this paper, we proposed a novel approach utilizing the multiple functions of the BG, which resulted in a hydrogel with both tissue adhesive and bioactive properties. Our results demonstrated that the BG/OSA composite hydrogel was not only adhesive enough for wound closure in vivo but also stimulated angiogenesis in vitro and enhanced in vivo blood vessel formation during wound healing.

SA is a biocompatible natural polymer that is suitable for the preparation of hydrogels through chemical crosslinking after periodate oxidation ${ }^{25,29}$. However, the OSA hydrogel obtained by the conventional cross-linking method has limited adhesive ability to tissues because the $\mathrm{pH}$ value in the wound area is generally in the weakly acid or neutral range $(5.4-7.4)^{13}$ or even acidic in cancer tissues ${ }^{14}$, which does not favor the adhesive interaction between the hydrogel and tissues. A weak alkaline environment is required for amide formation between the hydrogel and tissues ${ }^{10}$. Therefore, we utilized the function of BG in our material design, in which the BG particles slowly released alkaline ions to create a mild alkaline environment in favor of the amide-forming reaction. The results confirmed the effectiveness of our design and demonstrated that the introduction of BG significantly improved the tissue-bonding ability of the OSA hydrogel and the BG/OSA hydrogel provided complete wound closure in vivo. Moreover, our BG/OSA composite hydrogel not only showed high tissue adhesiveness but also revealed good adhesiveness to different medical implantable materials, such as titanium alloy, silicon, and calcium phosphate bioceramics.

In comparison with the pure OSA hydrogel, the increased adhesiveness of the BG/OSA hydrogel to materials can be explained by the enhanced viscosity of the hydrogel, which was caused by the physical crosslinking via chelation between the $\mathrm{Ca}^{2+}$ released from the 
A
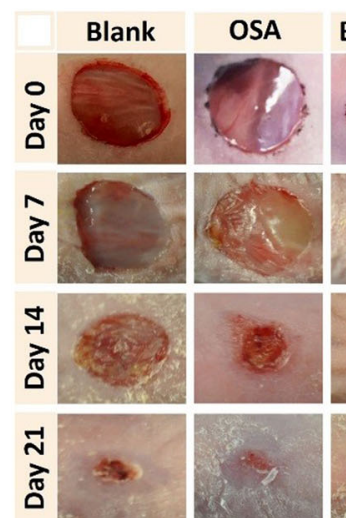

C

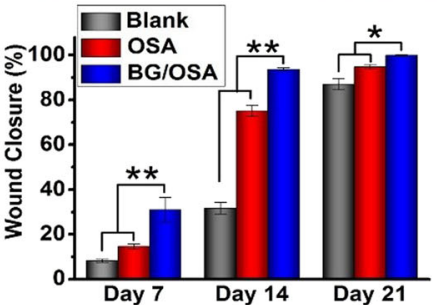

B
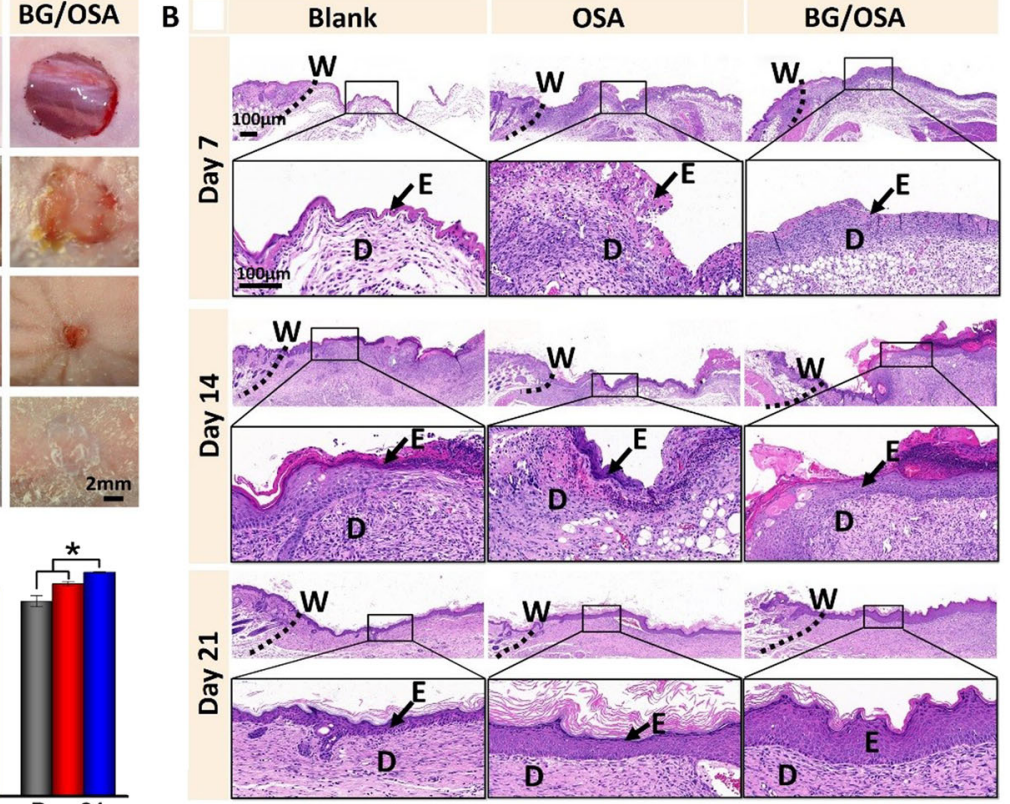

Fig. 6 Enhanced wound healing by the bioactive BG/OSA hydrogel. a Wound-healing process 0, 7, 14, and 21 days after OSA hydrogel and 1.0\%BG/OSA composite hydrogel treatment (Blank: the group without any treatment). $\mathbf{b}$ H\&E staining of wound sections after 7, 14, and 21 days with OSA hydrogel and 1.0\%-BG/OSA composite hydrogel treatment (E: epidermis, D: dermis, W: wound edge). c Quantification of the wound closure and newly formed epidermal tissue
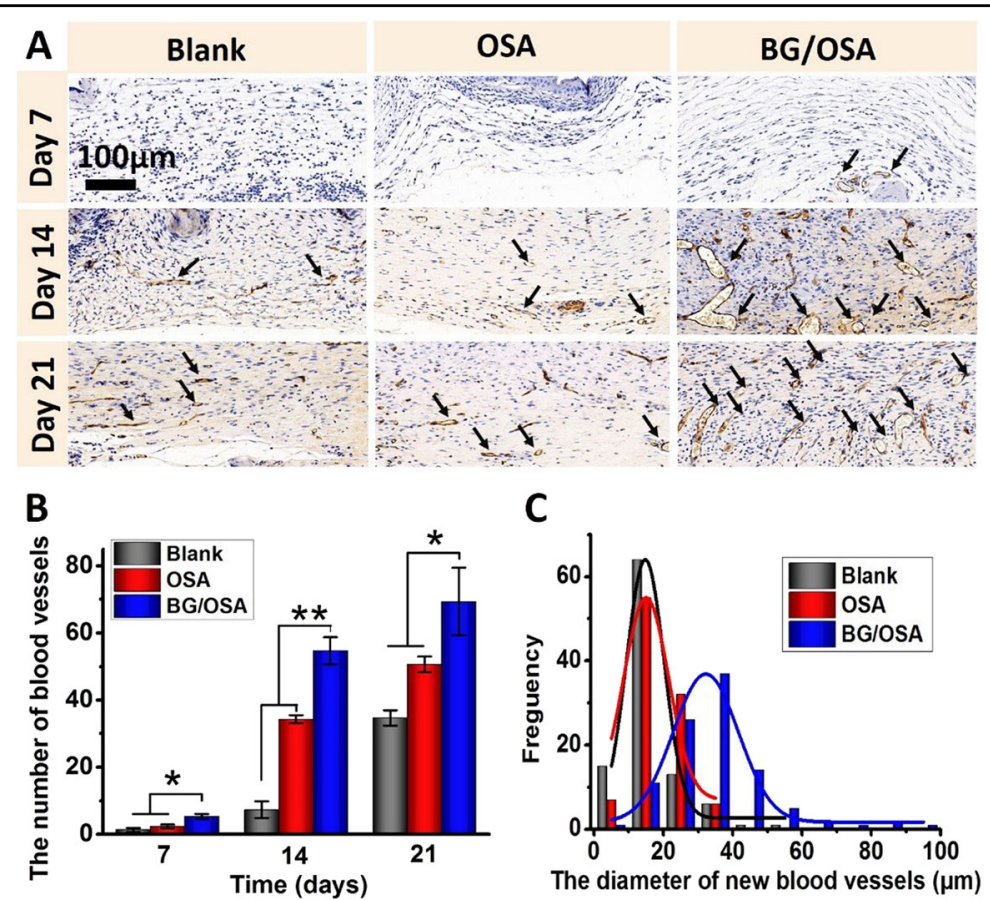

C

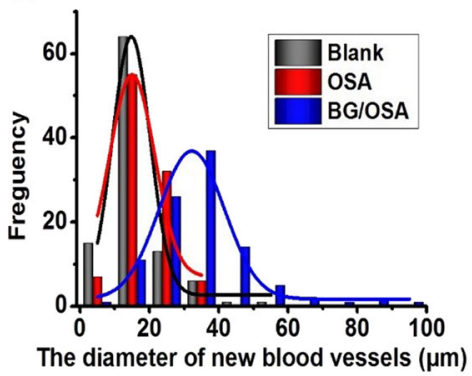

Fig. 7 Enhanced angiogenesis by the BG/OSA composite hydrogel during the wound-healing process. a Immunohistochemical staining of CD 31 in sections on days 7, 14, and 21 (black arrows indicate the blood vessels). $\mathbf{b}$ The number of newly formed blood vessels measured from the immunohistochemical images. $\mathbf{c}$ The diameter of the newly formed blood vessels on day 21 measured from the immunohistochemical images. The data represent the means $\pm \mathrm{SD}(n=100) .\left({ }^{*} P<0.05,{ }^{* *} P<0.01\right)$ 


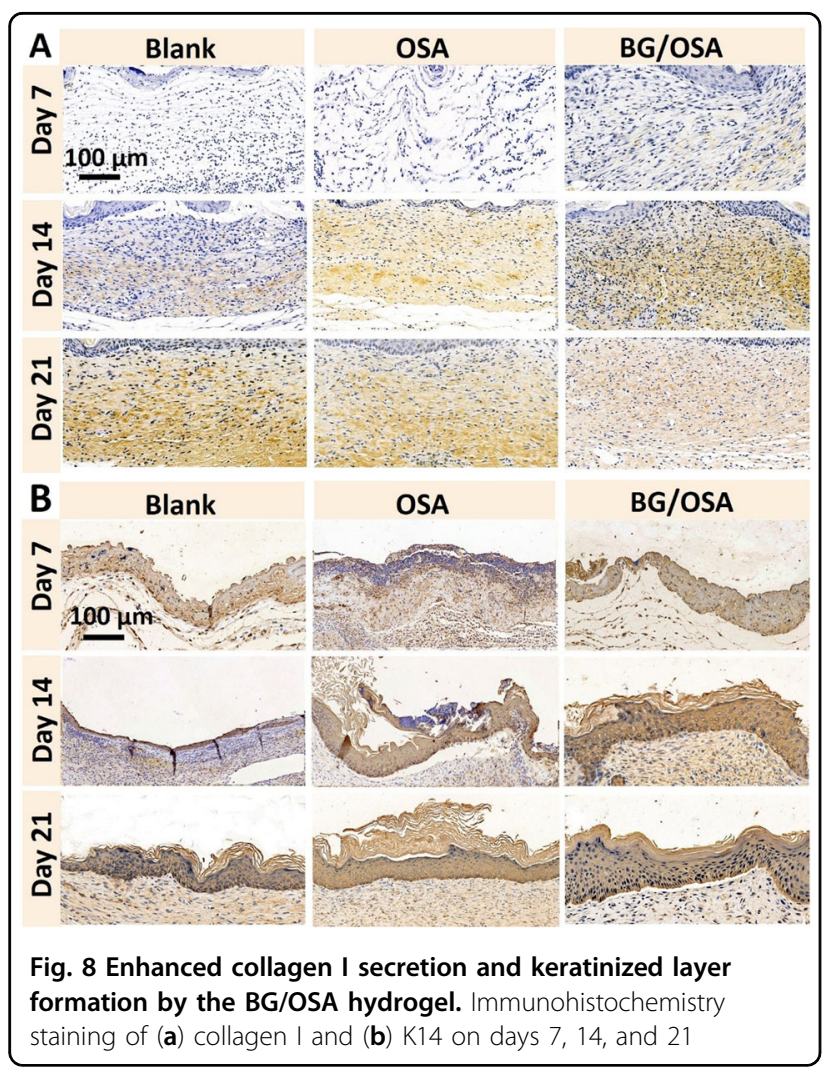

BG and the carboxyl groups of the polymer chains of the hydrogel. By comparing the adhesiveness and viscosities of the BG/OSA hydrogel with the CA/OSA and $\mathrm{Na}_{2} \mathrm{CO}_{3} /$ OSA hydrogels, we can conclude that $\mathrm{Ca}$ ions are the main factor determining the adhesiveness of the hydrogel to the material. Furthermore, the low adhesiveness and viscosity of the $\mathrm{CaCl}_{2} / \mathrm{OSA}$ hydrogel suggest that the sustained release of $\mathrm{Ca}$ ions from $\mathrm{BG}$ or calcium carbonate particles is critical for chelation cross-linking. Direct addition of the $\mathrm{CaCl}_{2}$ solution results in the rapid reaction between $\mathrm{Ca}^{2+}$ and the guluronate blocks of alginate chains, which does not favor uniform gel formation and significantly affects the hydrogel properties. Moreover, the increased adhesiveness of BG/OSA might also be partially explained by the interlock effect, in which a rougher surface and a more viscous adhesive enable easier fluid penetration, producing more mechanical interlock joints and hence better adhesion ${ }^{16,17}$. All of this evidence demonstrated that BG played a multifunctional role in the BG/OSA composite hydrogel due to its unique dualadhesive property, which was not only adhesive to tissues but also to implantable materials.

Another important property of hydrogels for woundhealing applications is their bioactivity to wound-dressing materials to enhance the healing process. Incorporating the BG particles into hydrogel systems will endow the hydrogel with bioactivity for enhanced angiogenesis and

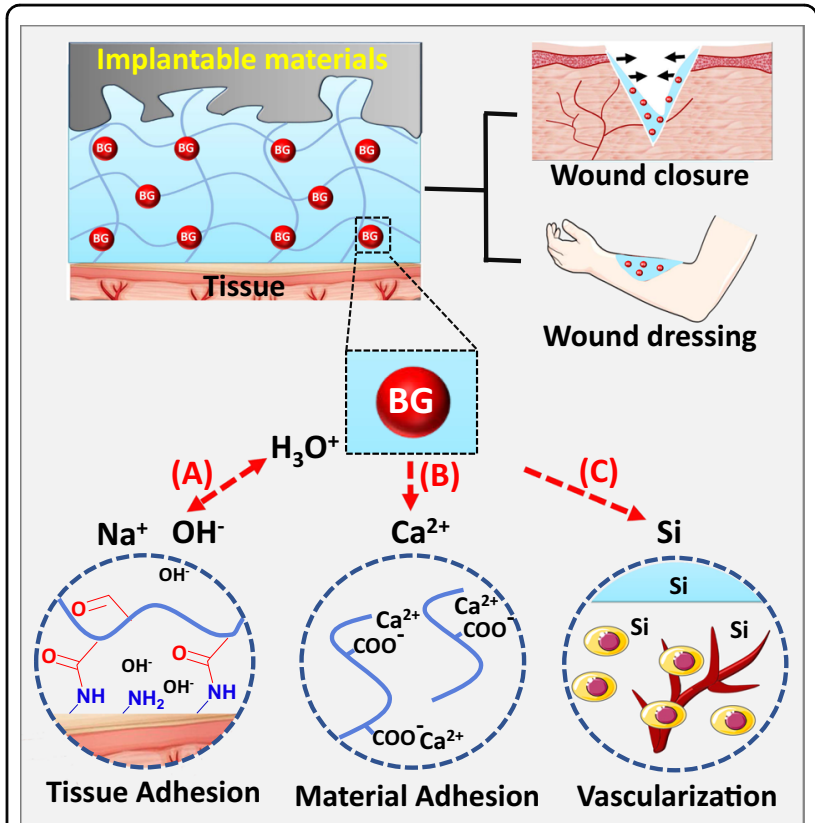

Fig. 9 Summary of the dual-adhesive and bioactive BG/OSA hydrogels. a The rapid ion exchange on the BG surface makes an alkaline environment to promote imine linkage formation between the tissues and the hydrogel. $\mathbf{b}$ The BG promotes adhesion between the hydrogel and materials via chelation between the carboxyl groups and $\mathrm{Ca}^{2+}$ released from BG. c The released $\mathrm{Si}$ ions promote vascularization in the wound area

wound healing by releasing $\mathrm{Si}$ ions ${ }^{21,26}$. Here, we also confirmed that in our novel adhesive hydrogel system, BG maintained its bioactivity and endowed the composite hydrogel with excellent activity to stimulate wound healing by enhancing angiogenesis. According to all the results obtained, we confirmed our hypothesis that BG plays multiple roles in the formation of the bioactive and dual-adhesive hydrogel as a bifunctional material for wound healing. The possible mechanisms of the BG/OSA composite hydrogel for the formation of adhesiveness to both tissues and implantable materials and the bioactivity to stimulate wound healing are illustrated in Fig. 9. First, the rapid ion exchange on the BG surface creates an alkaline environment, which accelerates the formation of an imine bond for enhanced adhesiveness of the hydrogel to the tissues. Second, the release of $\mathrm{Ca}^{2+}$ from the BG chelates with the carboxyl groups in the OSA and $\gamma$-PGA chains to endow the hydrogel with adhesiveness to implantable materials. Furthermore, BG releases $\mathrm{Si}$ ions, which enhances angiogenesis and accelerates the wound healing process in vivo.

\section{Conclusion}

In summary, we developed a novel dual-adhesive and bioactive BG/OSA hydrogel for wound closure and 
enhancement of wound healing. The adhesiveness of the OSA hydrogel to tissues was significantly enhanced by the introduction of BG with the release of alkaline ions, which was confirmed by the wound closure test in vivo. Furthermore, the adhesive hydrogel showed good adhesiveness to different implantable biomaterials due to chelation of $\mathrm{Ca}$ ions with the carboxyl groups in the hydrogel matrix, which might be useful for preventing implant dislocation. In addition to the dual adhesiveness to both tissues and implantable materials, the BG/OSA hydrogel has also been demonstrated to be bioactive to stimulate angiogenesis and promote chronic wound healing in vivo.

\section{Acknowledgements}

This work was supported by the National Key Research and Development Plan of China (No. 2016YFC1100201).

\section{Author details}

${ }^{1}$ State Key Laboratory of High Performance Ceramics and Superfine Microstructure, Shanghai Institute of Ceramics, Chinese Academy of Sciences, 1295 Dingxi Road, Shanghai 200050, China. ${ }^{2}$ Center of Materials Science and Optoelectronics Engineering, University of Chinese Academy of Sciences, 19 Yuquan Road, Beijing 100049, China. ${ }^{3}$ School of Pharmacy, Shanghai Jiao Tong University, 800 Dongchuan Road, Shanghai 200240, China

\section{Conflict of interest}

The authors declare that they have no conflict of interest.

\section{Publisher's note}

Springer Nature remains neutral with regard to jurisdictional claims in published maps and institutional affiliations.

Supplementary information is available for this paper at https://doi.org/ 10.1038/s41427-019-0168-0.

Received: 20 June 2019 Revised: 29 August 2019 Accepted: 4 September 2019.

Published online: 15 November 2019

\section{References}

1. Peppas, A. N., Hilt, J. Zach, Khademhosseini, Ali \& Langer, R. Hydrogels in biology and medicine: from molecular principles to bionanotechnology. Adv. Mater. 18, 1345-1360 (2006).

2. Bouten, Petra. J. M. et al. The chemistry of tissue adhesive materials. Prog. Polym. Sci. 39, 1375-1405 (2014).

3. Duartea, A. P., Coelhoa, J. F., Bordadob, J. C., Cidadec, M. T. \& Gil, M. H. Surgical adhesives: systematic review of the main types and development forecast. Prog. Polym. Sci. 37, 1031-1050 (2012).

4. Ghobril, C. \& Grinstaff, M. W. The chemistry and engineering of polymeric hydrogel adhesives for wound closure: a tutorial. Chem. Soc. Rev. 44, 1820-1835 (2015).

5. Yu, Y. et al. Multifunctional "hydrogel skins" on diverse polymers with arbitrary shapes. Adv. Mater. 31, 1807101 (2019).

6. Deng, Z. et al. Stimuli-responsive conductive nanocomposite hydrogels with high stretchability, self-healing, adhesiveness, and 3D printability for human motion sensing. ACS Appl. Mater. interfaces 11, 6796-6808 (2019).

7. Eriksen, J. R., Bech, J. I., Linnemann, D. \& Rosenberg, J. Laparoscopic intraperitoneal mesh fixation with fibrin sealant (Tisseel) vs. titanium tacks: a randomised controlled experimental study in pigs. Hernia: J. Hernias Abdom. Wall Surg. 12, 483-491 (2008).

8. Lu, H. et al. Tough, self-healable and tissue-adhesive hydrogel with tunable multifunctionality. NPG Asia Mater. 9, 1-12 (2017).
9. Xin, L. et al. Hydrogels tackified by nucleobases. Adv. Funct. Mater. 27, 1703132-1703137 (2017).

10. $\mathrm{Yu}, \mathrm{W}$. et al. A soft tissue adhesive based on aldehyde-sodium alginate and amino-carboxymethyl chitosan preparation through the Schiff reaction. Front Mater. Sci. 11, 215-222 (2017).

11. Zhao, W. et al. Novel biocompatible polysaccharide-based self-healing hydrogel. Adv. Funct. Mater. 25, 1352-1359 (2015).

12. Zeng, Q. et al. Self-healing elastin-bioglass hydrogels. Biomacromolecules $\mathbf{1 7}$, 2619-2625 (2016)

13. Dissemond, J., Witthoff, M., Brauns, T. C., Haberer, D. \& Goos, M. pH values in chronic wounds:evaluation during modern wound therapy. Der Hautarzt; Z fur Dermatologie, Venerologie, und Venwandte-. Geb. 54, 959-665 (2003).

14. Rofstad, E. K., Mathiesen, B., Kindem, K. \& Galappathi, K. Acidic extracellular pH promotes experimental metastasis of human melanoma cells in athymic nude mice. Cancer Res. 66, 6699-6707 (2006).

15. Han, Y., Zeng, Q., Li, H. \& Chang, J. The calcium silicate/alginate composite: preparation and evaluation of its behavior as bioactive injectable hydrogels. Acta Biomaterialia. 9, 9107-9117 (2013).

16. Yang, J., Bai, R., Chen, B. \& Suo, Z. Hydrogel adhesion: a supramolecular synergy of chemistry, topology, and mechanics. Adv. Funct. Mater. 29, 1901693 (2019).

17. Han, L. et al. Mussel-inspired tissue-adhesive hydrogel based on the polydopamine-chondroitin sulfate complex for growth-factor-free cartilage regeneration. ACS Appl. Mater. Interfaces 10, 28015-28026 (2018).

18. Yan, H. et al. Injectable bioactive akermanite/alginate composite hydrogels for in situ skin tissue engineering. J. Mater. Chem. B 5, 3315-3326 (2017).

19. Li, Y. et al. Multifunctional hydrogels prepared by dual ion cross-linking for chronic wound healing. ACS Appl. Mater. Interfaces 9, 16054-16062 (2017).

20. Xing, M., Wang, X., Wang, E., Gao, L. \& Chang, J. Bone tissue engineering strategy based on the synergistic effects of silicon and strontium ions. Acta Biomater. 72, 381-395 (2018).

21. Zhou, Y. et al. Bioglass activated albumin hydrogels for wound healing. Adv Healthc. Mater. 7, 1800144 (2018).

22. Joo, S., Ko, I. K., Atala, A., Yoo, J. J. \& Lee, S. J. Amniotic fluid-derived stem cells in regenerative medicine research. Arch. Pharm. Res. 35, 271-280 (2012).

23. Pratt, A. B., Weber, F. E., Schmoekel, H. G., Muller, R. \& Hubbell, J. A. Synthetic extracellular matrices for in situ tissue engineering. Biotechnol. Bioeng. 86, 27-36 (2004).

24. Zhang, X. et al. Stimulation of wound healing using bioinspired hydrogels with basic fibroblast growth factor (bFGF). Int. J. Nanomed. 13, 3897-3906 (2018).

25. Reakasame, S. \& Boccaccini, A. R. Oxidized alginate-based hydrogels for tissue engineering applications: a review. Biomacromolecules 19, 3-21 (2018).

26. $Y u, H .$, Peng, J., Xu, Y., Chang, J. \& Li, H. Bioglass activated skin tissue engineering constructs for wound healing. ACS Appl. Mater. Interfaces 8, 703-715 (2016).

27. Brauer, D. S. Bioactive glasses-structure and properties. Angew. Chem. -Int. Ed. 2015, 4160-4181 (2015).

28. Li, A. et al. Mineral-enhanced polyacrylic acid hydrogel as an oyster-inspired organic-inorganic hybrid adhesive. ACS Appl. Mater. Interfaces 10, 10471-10479 (2018).

29. Yan, S. et al. Injectable in situ self-cross-linking hydrogels based on poly (Lglutamic acid) and alginate for cartilage tissue engineering. Biomacromolecules 15, 4495-4508 (2014)

30. Yan, S. et al. Injectable in situ forming poly(l-glutamic acid) hydrogels for cartilage tissue engineering. J. Mat. Chem. B 4, 947-961 (2016).

31. Lal, N., Jennifer, Monahan, Richard, L., Stroshine, Jonathan, J., Wilker \& Shi, R. Adhesive strength of marine mussel extracts on porcine skin. Biomaterials 24, 4091-4099 (2003)

32. $\mathrm{Xu}, \mathrm{Y}$., Liang, K., Ullah, W., Ji, Y. \& Ma, J. Chitin nanocrystal enhanced wet adhesion performance of mussel-inspired citrate-based soft-tissue adhesive. Carbohydr. Polym. 190, 324-330 (2018).

33. Wang, X. et al. Silicon-enhanced adipogenesis and angiogenesis for vascularized adipose tissue engineering. Adv. Sci. 5, 1800776 (2018).

34. Meddahi-Pelle, A. et al. Organ repair, hemostasis, and in vivo bonding of medical devices by aqueous solutions of nanoparticles. Angew. Chem. -Int. Ed. 53, 6369-6373 (2014).

35. Yan, S. et al. Injectable in situ forming poly(l-glutamic acid) hydrogels for cartilage tissue engineering. J. Mat. Chem. B 4, 947-961 (2016). 
36. Qu, J. et al. Antibacterial adhesive injectable hydrogels with rapid self-healing, extensibility and compressibility as wound dressing for joints skin wound healing. Biomaterials 183, 185-199 (2018).

37. Liao, M. et al. Wearable, healable, and adhesive epidermal sensors assembled from mussel-inspired conductive hybrid hydrogel framework. Adv. Funct. Mater. 27, 1703852 (2017).

38. Liang, Y. et al. Adhesive hemostatic conducting injectable composite hydrogels with sustained drug release and photothermal antibacterial activity to promote full-thickness skin regeneration during wound healing. Small $\mathbf{1 5}$ 1900046 (2019).

39. Qu, J. et al. Degradable conductive injectable hydrogels as novel antibacterial, anti-oxidant wound dressings for wound healing. CHEM ENG J. 362, 548-560 (2019).

40. Zhao, X. et al. Antibacterial anti-oxidant electroactive injectable hydrogel as self-healing wound dressing with hemostasis and adhesiveness for cutaneous wound healing. Biomaterials 122, 34-47 (2017). 